\title{
KARL MARX ÜBER DAS BAUERNTUM UND DIE BÜNDNISFRAGE
}

$\mathrm{Zu}$ dem theoretischen und politischen Erbteil, das Karl Marx der Arbeiterbewegung hinterlassen hat, gehört die Lehre von dem Bündnis zwischen Proletariat und Bauerntum, die für die marxistischen Parteien bis in die Gegenwart von Bedeutung geblieben ist. Ihre erste Bewährungsprobe hat sie schon am Ende des 19. Jahrhunderts bestehen müssen, als die deutsche Sozialdemokratie vor der Frage gestanden hat, ob sie in programmatischer Form für die Bauern eintreten solle oder nicht. ${ }^{1}$ Ihre 1895 auf dem Parteitag von Breslau ausgesprochene Absage an ein bauernfreundliches Agrarprogramm hat das Dilemma offenbart, das dem Marxschen Konzept der Bündnisfrage innewohnte: das Dilemma zwischen der politischen Notwendigkeit des Bündnisses und der theoretischen Bedenklichkeit der dafür einzusetzenden Mittel. Einerseits war es für die Arbeiterparteien erstrebenswert, ihren politischen Einflußbereich über die engeren sozialen Grenzen, die ihnen gezogen waren, auszuweiten, aber andererseits hatten sie dabei die sozialistischen Prinzipien zu berücksichtigen, die das Ziel ihres politischen Strebens festlegten. Das bedingte, daß das Zusammengehen mit den Bauern nicht um jeden Preis, sondern nur zu einem theoretisch zulässigen herbeigeführt werden durfte und daß die Arbeiterparteien zu entscheiden hatten, welche Politik gegenüber den Bauern mit marxistischen Grundsätzen vereinbar war und welche Maßnahmen am ehesten die Aussicht boten, einen Teil der Landbevölkerung für die eigene Sache interessieren zu können.

Es lag in der Natur der Dinge, daß diese Frage, die von Marx zu einem wesentlichen Element der Strategie und Taktik einer proletarischen Revolution erhoben worden war, von den Arbeiterparteien nach den Aussagen der Marxschen Lehre beantwortet werden sollte, und es ist deshalb geboten zu prüfen, was Marx von der ökonomi-

\footnotetext{
1. Vgl. die ungedruckten Dissertationen von G. Dittmar, Zur Theorie und der Praxis der sozialdemokratischen Landagitation unter den deutschen Kleinbauern in den goer Jahren des 19. Jahrhunderts, phil. Diss. Rostock $1964 ; \mathrm{H}$. Hesselbarth, Die deutsche Sozialdemokratie und die Bauern ( 1890 bis 1895 ), phil. Diss. Berlin (Ost) 1964; H. Nau, Sozialdemokratische Agrarpolitik und Bauernschaft, agr. Diss. Berlin (Ost) 1961 .
} 
schen und sozialen Rolle der Bauern in der kapitalistischen Gesellschaft gehalten hat und welche Prämissen er dem Bündnis zwischen Proletariern und Bauern zugrundegelegt hat. Da es eine systematische Abhandlung der Bauernfrage aus der Feder Marx' nicht gibt, müssen die einschlägigen Bemerkungen aus den verschiedenen Teilen des Gesamtwerks ermittelt und miteinander kombiniert werden, ${ }^{1}$ wobei eine Beschränkung auf die für die Bündnisfrage wesentlichen Gesichtspunkte angestrebt wird. Unter Bündnis ist das gemeinsame politische Vorgehen des Proletariats mit demjenigen Teil des Bauerntums zu verstehen, dessen sozial-ökonomische Interessen als mit den spezifischen Zielen des Proletariats vereinbar erscheinen konnten; und die Bündnisfrage beinhaltet demnach das Problem, wie das Bündnis im Einzelfall zu realisieren sei.

Eine Beschäftigung mit dem Bauerntum ist für Karl Marx unter zwei Gesichtspunkten denkbar gewesen: unter dem theoretischen und dem politischen. Im ersteren Falle wäre es darum gegangen, die Stellung des Bauerntums in der modernen Gesellschaft zu erkennen sowie daraus auf seine künftige Rolle zu schließen; im zweiten Falle wäre es die Aufgabe gewesen, das politisch-praktische Verhalten der Arbeiterklasse gegenüber dem in seinem Wesen erkannten Bauerntum zu bestimmen. Was den theoretischen Aspekt betrifft, so hat Marx dem Bauerntum in dieser Hinsicht kein besonderes Interesse entgegengebracht. Es ist ihm eine dem Mittelalter adäquate Wirtschaftsform gewesen, die im Laufe der Neuzeit vom weitfächigen Großbetrieb verdrängt worden sei bzw. noch verdrängt werden sollte. ${ }^{2}$ Infolgedessen hat er bei der Analyse der kapitalistischen Landwirtschaft - und mit dieser allein hat er sich theoretisch beschäftigt - nicht vom bäuerlichen Betrieb, sondern lediglich vom Großbetrieb gesprochen, genauer gesagt: vom Pachtbetrieb, wie er für das damalige England kennzeichnend gewesen ist. ${ }^{3}$ An diesem speziellen Objekt studierte

\footnotetext{
${ }^{1}$ Einen relativ ausführlichen Uberblick bietet F. Schaaf, Der Kampf der deutschen Arbeiterbewegung um die Landarbeiter und werktätigen Bauern I 848-189o, Berlin 1962 , S. 2gff. Von H. Hesselbarth, Blatt 304f., ist auf das Fehlen einer zusammenhängenden Darstellung der Marxschen Theorie von den Bauern hingewiesen, die Existenz solch einer Theorie aber als unbestreitbar angesehen worden.

2 Die Wege, auf denen sich der Großbetrieb an die Stelle des Bauernbetriebs gesetzt hatte, hat Marx im I. Band des „Kapital” beschrieben, s. K. Marx und F. Engels, Werke [= MEW], Bd. XXIII, S. 744ff.

3 Marx' Agrartheorie, deren Kern die Lehre von der Differentialrente und der absoluten Rente ist - enthalten im 3. Band des "Kapital”, s. MEW XXV, S. 653 ff., $756 \mathrm{ff}$. -, ist in folgenden Werken bereits dargestellt worden: K. Kautsky, Die Agrarfrage, Stuttgart
} 
er die Wirkungen des Kapitalismus in der Landwirtschaft und von hier aus gelangte er zu der Erkenntnis, daß die kapitalistische Landwirtschaft in einen vollendeten Raubbau an Mensch und Boden ausarten werde, den nur die proletarische Revolution beseitigen könne. Das sollte geschehen durch die Aufhebung des Privateigentums an Grund und Boden, da dieses „,der agrikolen Produktion und der rationellen Behandlung, Erhaltung und Verbesserung des Bodens selbst" ein Hindernis entgegensetzte. ${ }^{1}$ Das siegreiche Proletariat hatte die ländlichen Verhältnisse ,auf Grundlage [...] der Kooperation und des Gemeinbesitzes der Erde und der durch die Arbeitskraft selbst produzierten Produktionsmittel" neu zu gestalten. ${ }^{2}$ Grund und Boden sollten nationalisiert werden, ${ }^{3}$ wobei auch zugunsten des genossenschaftlichen Eigentums keine Ausnahme gemacht werden durfte, da es sonst dahin kommen könnte, daß „die ganze Gesellschaft einer besonderen Klasse [genossenschaftlicher] Produzenten" ausgeliefert würde. ${ }^{4}$ So erhob sich hier hinter der Entfaltung des Kapitalismus auf dem Lande ebenso wie in der Industrie das Endziel einer sozialistischen Wirtschaftsform.

Dieses Endziel galt für die gesamte Landwirtschaft, d.h. für Großund Kleinbetriebe. Aber während die Großbetriebe dieses Stadium durch ihre eigene Entwicklung vorbereiteten, trieben die Kleinbetriebe nur noch passiv in der Strömung, da sie nicht mehr die Kraft besaßen, nach außen wirkende Impulse auszustrahlen. Sie sind zwar „für die Entwicklung der Agrikultur selbst ein notwendiger Durchgangspunkt" gewesen, ${ }^{5}$ aber das lag lange zurück; in der Gegenwart drohte ihnen nur noch der Untergang, da die kapitalistische Produktionsweise in der Landwirtschaft „das Bollwerk der alten Gesellschaft vernichtet, den ,Bauer', und ihm den Lohnarbeiter unterschiebt", also den Bauern seines Grundeigentums beraubte und zum Proletarier degradierte. Wenn die bäuerlichen Kleinbetriebe trotzdem noch nicht restlos verschwunden waren, so hatte das seine Ursache darin,

1899, S. 56ff.; W. Cohnstaedt, Die Agrarfrage in der deutschen Sozialdemokratie von Karl Marx bis zum Breslauer Parteitag, München I903, S. Ioff.; M. Kemper, Marxismus und Landwirtschaft. Eine Darstellung der Entwicklungstendenzen marxistischer Agrartheorie und Agrarpolitik, agr. Diss. Bonn-Poppelsdorf I929, S. 13ff.; vgl. auch A. Benary, Aktuelle Probleme der Agrartheorie des Marxismus-Leninismus, Berlin ig5s, S. I gff.; K.

Ritter, Agrarwirtschaft und Agrarpolitik im Kapitalismus, x. Halbband, Berlin I955, S. $95 \mathrm{ff}$.

1 MEW XXV, S. 821.

MEW XXIII, S. 79 1.

"MEW XVIII, S. 6off. („Uber die Nationalisierung des Grund und Bodens”, 1872).

A.a.O., S. 62.

5 MEW XXV, S. 81s; vgl. MEW III, S. 33r f., MEW XVI, S. 598.

- MEW XXIII, S. s28. 
daß die kapitalistische Produktionsweise nur „langsam und ungleichmäßig die Landwirtschaft" ergriff und daß die zögernde „Verwandlung der selbstwirtschaftenden Bauern in Lohnarbeiter" hier zu gröBeren Ungleichheiten führte als in irgendeinem andern Wirtschaftszweig. ${ }^{1}$ Die Landwirtschaft hinkte bei der Ausbildung rein kapitalistischer Produktionsverhältnisse hinter der übrigen Wirtschaft her, so daß sich in ihr nicht-kapitalistische Existenzen länger als sonst üblich behaupten konnten. Das traf vor allem für die kleinen und mittleren Bauern zu, die von ihrer eigenen Hände Arbeit lebten und in erster Linie nicht auf Profit, sondern auf Deckung ihres Lebensunterhaltes ausgingen. ${ }^{2}$ Für sie war es kennzeichnend, daß bei ihnen Besitz der Produktionsmittel und Arbeit noch vereinigt waren, ${ }^{3}$ sie also in einer vorkapitalistischen Form des Produktionsverhältnisses lebten, in der der spätere Gegensatz zwischen Kapitalist und Lohnarbeiter noch nicht entwickelt war. Diese vorkapitalistische Qualität des Bauern stellte aber unter den modernen Verhältnissen keinen wirtschaftlichen oder sozialen Vorteil dar, da sie an sich ,nur verträglich [sei] mit engen naturwüchsigen Schranken der Produktion und $\mathrm{Ge}$ sellschaft",4 mithin die "Bedingungen einer rationellen Kultur" ausschließe. ${ }^{5}$ Das Hauptübel der kleineren Bauern erblickte Marx in deren Kapitalschwäche, ${ }^{6}$ die es ihnen verwehrte, sich der Mittel zu bedienen, die den Aufschwung der Arbeitsproduktivität bedingten. Die Folge davon war, daß durch Mehraufwand an Arbeit ausgeglichen werden mußte, was andernorts durch den Einsatz von Kapital und Technik erreicht wurde. „Es ist richtig, daß z.B. der Bauer auf seine kleine Parzelle viel Arbeit verwendet. Aber isolierte und der objektiven, sowohl der gesellschaftlichen wie der materiellen Bedingungen der Produktivität beraubte, von ihnen entblößte Arbeit".? Hier herrschten ,unendliche Zersplitterung der Produktionsmittel und Vereinzelung der Produzenten selbst. Ungeheure Verschwendung von Menschenkraft",

1 MEW XXV, S. 689, 663.

2 A.a.O., S. 814, 703; vgl. D. Mitrany, Marxismus und Bauerntum, München 1956, S. 22.

${ }^{3}$ MEW XXV, S, 627: bei der kleinbäuerlichen Agrikultur sei „der Besitz von Grund und Boden [...] eine der Produktionsbedingungen für den unmittelbaren Produzenten". Vgl. S. 8I 5; ferner MEW XXIII, S. 789: „Das Privateigentum des Arbeiters an seinen Produktionsmitteln ist die Grundlage des Kleinbetriebes, der Kleinbetrieb eine notwendige Bedingung für die gesellschaftliche Produktion." Vgl. D. Mitrany, S. 22.

4 MEW XXIII, S. 789 .

5 MEW XXV, S. 821 .

- A.a.O., S. 8r6ff. Der Kapitalmangel zog den Zwang zur Verschuldung nach sich, so $\mathrm{da} B$ das verpfändete bäuerliche Grundeigentum de facto zu einen Eigentum der Gläubiger wurde.

7 A.a.O., S. 689 .

8 A.a.O., S. 81 sf.; vgl. MEW XXIII, S. 789. 
tation und Vergeudung der Bodenkräfte", ${ }^{1}$ so daß die wirtschaftliche Leistungsfähigkeit weit hinter den natürlichen und gesellschaftlichen Möglichkeiten zurückblieb. Diese Beschränktheit prägte auch die soziale Lage des Bauern, da eine zu extremer und unproduktiver Kraftanstrengung gezwungene Klasse außerstande war, an den Errungenschaften der gesellschaftlichen und kulturellen Entwicklung teilzunehmen. Das führte dahin, daß ,das kleine Grundeigentum eine halb außerhalb der Gesellschaft stehende Klasse von Barbaren schafft, die alle Rohheit primitiver Gesellschaftsformen mit allen Qualen und aller Misere zivilisierter Länder verbindet".2

\section{II}

So betrachtet bot das Bauerntum inmitten der kapitalistischen Gesellschaft den Anblick einer zum Elend verurteilten Schicht dar, weil seine ökonomischen und geistigen Mittel nicht ausreichten, den Anschluß an die moderne Entwicklung zu gewinnen. Das mochte den Anschein erwecken, als befänden sich die Bauern in einer Situation, die sie dazu prädestinierte, der Bundesgenosse des Proletariats zu werden, da beide nur Ausbeutung und Verelendung vom kapitalistischen System zu erwarten hatten. Doch Marx beurteilte das anders. Obwohl er nicht verkannte, daß die Bauern ähnlich wie andere Schichten des Mittelstandes gegen das kapitalistische Großbürgertum ankämpften, vermißte er hierbei die Identität mit den Bestrebungen des Proletariats. Während dieses die Heilung der sozialen Gebrechen von der Aufhebung des Privateigentums an den Produktionsmitteln erwartete, setzten sich die Bauern dafür ein, das kleine Bodeneigentum als Grundlage ihres Daseins zu behaupten. Sie lehnten nicht das Privateigentum als solches, sondern nur dessen Konzentration ab; das machte sie zu Verteidigern eines überholten Zustandes, nämlich des Zustandes der vorkapitalistischen Zersplitterung der Produktionsmittel. „Die Mittelstände, der kleine Industrielle, der kleine Kaufmann, der Handwerker, der Bauer, sie alle bekämpfen die Bourgeoisie, um ihre Existenz als Mittelstand vor dem Untergange zu sichern. Sie sind also nicht revolutionär, sondern konservativ. Noch mehr, sie

1 MEW XXV, S. 820.

2 A.a.O., S. 82 r. Vgl. auch die Wendung vom „Idiotismus des Landlebens" im Kommunistischen Manifest, MEW IV, S. 466. Nicht leicht zu interpretieren ist in diesem Zusammenhange die günstige Beurteilung des Kleinbauern, die Marx bei Gelegenheit der Wirkungen, die der Zyklus von Preissteigerung und Uberproduktion auf den Fortschritt der landwirtschaftlichen Produktion hatte, einfließen ließ und derzufolge "die rationelle Agrikultur unverträglich ist mit dem kapitalistischen System [...] und entweder der Hand des selbst arbeitenden Kleinbauern oder der Kontrolle der assoziierten Produzenten bedarf". MEW XXV, S. I3I. 
sind reaktionär, denn sie suchen das Rad der Geschichte zurückzudrehen." I Die wahren Ursachen seiner Misere verkennend, hänge der Bauer ,mit blinder Liebe an seinem Stückchen Erde und seinem bloß nominellen Besitzrecht”, so daß er in einen „höchst verhängnisvollen Gegensatz zur Industriearbeiterklasse” gerate und „zum entschiedensten Feind jeden gesellschaftlichen Fortschritts und vor allem der Nationalisierung des Grund und Bodens" werde. ${ }^{2}$ Deshalb war es bedenklich, auf die speziellen Bedürfnisse der Bauern einzugehen, denn sie mußten mit den sozialistischen Prinzipien der Arbeiterklasse kollidieren; solange die Bauern auf ihrem Privateigentum bestanden, war es unvermeidlich, daß sich zwischen ihnen und den Industriearbeitern eine Kluft auftat.

Dieser Erkenntnis, die zu einer zurückhaltenden Beurteilung der Bündnismöglichkeiten mahnte, stand jedoch die Erfahrung gegenüber, daß sich die Arbeiterpartei bei einem Verharren auf diesem Standpunkt der Gefahr aussetzte, für alle Zeiten eine isolierte Minderheit in der Gesellschaft zu bleiben. Was das im Ernstfalle bedeutete, hatte die Revolution von 1848 gezeigt, wo das sich selbst überlassene Proletariat den Machtmitteln der herrschenden Klassen nicht hatte standhalten können. Diese Erfahrung ist Marx durchaus gegenwärtig gewesen und sie hat ihn bewogen, einen Ausweg aus dem Dilemma zu suchen. Der vergebliche Kampf der Pariser Arbeiter im Jahr I848 hatte ihm eröffnet, daß der bürgerlichen Ordnung nicht beizukommen war, solange „der Gang der Revolution die zwischen dem Proletariat und der Bourgeoisie stehende Klasse der Nation, Bauern und Kleinbürger, nicht gegen diese Ordnung, gegen die Herrschaft des Kapitals empört, sie gezwungen hatte, sich den Proletariern als ihren Vorkämpfern anzuschließen."3 1848 sei es so gewesen, daß dank dem allgemeinen Wahlrecht die Bauern zu Schiedsrichtern über das Schicksal Frankreichs geworden seien ${ }^{4}$ und daß sie sich zugunsten des Bonapartismus entschieden hätten. ${ }^{5}$ Infolge dieser Konstellation hing der Sieg des Proletariats von der Unterstützung durch die Bauern ab, die aber erst zu erwarten war, wenn die Bauern sich enttäuscht von dem bürgerlichen Regime abwenden und den blinden Glauben an ihr Privateigentum verlieren würden; dann erhalte „die proletarische Revolution das Chor, ohne das ihr Sologesang in allen Bauernnationen zum Sterbelied wird."6 Das Bündnis zwischen Arbeitern und

${ }^{1}$ MEW IV, S. 472 („Manifest der Kommunistischen Partei”, I848).

2 MEW XVIII, S. 6I (,Über die Nationalisierung" usw.).

${ }^{8}$ MEW VII, S. 2 I („Die Klassenkämpfe in Frankreich 1848 bis $1850 ”$, I 850 ).

4 A.a.O., S. I 8.

5 A.a.O., S. 44.

'MEW VII, S. 204, Anm. („Der achtzehnte Brumaire des Louis Bonaparte”, I8 2 ). 
Bauern war deshalb eine politische Notwendigkeit, der trotz prinzipieller Schwierigkeiten Rechnung getragen werden mußte. Marx glaubte, daß letzten Endes die kapitalistische Entwicklung dem Proletariat in diesem Punkte ungewollt zu Hilfe kommen werde. Denn mochten die Bauern auch der spezifischen inneren Qualität des Kapitalisten entbehren, so befreite sie das nicht davon, gewissermaßen auf Umwegen vom Kapitalismus ergriffen zu werden. Dieser konnte ihnen von außen her seine Gesetze aufzwingen, da sie sich den Anforderungen eines kapitalistisch umgestalteten Wirtschafts-, Gesellschafts- und Staatslebens nicht entziehen konnten. Die Bauern waren darauf angewiesen, einen Teil ihrer Erzeugnisse auf dem allgemeinen Markt abzusetzen, und hier herrschten die Gesetze der kapitalistischen Konkurrenz, die die Kleinproduzenten zum Tode verurteilten; sie mußten Steuern zahlen und vielfach Hypotheken verzinsen, was sie nötigte, Erträge zu erzielen, die über ihrem eigenen Lebensbedarf lagen. Dadurch wurden sie in die moderne Verkehrswirtschaft hineingerissen, der ihre produktiven Mittel nicht gewachsen waren. ${ }^{1}$ Das bewirkte, daß die Bauern, obwohl selbst keine Kapitalisten, vom Kapitalismus vernichtet wurden.

Nach Marx' Ansicht war ihre Lage bereits derart, daß sie keinen langen Widerstand mehr leisten konnten; ihr Eigentum war schon "seit langem aus seiner normalen Phase herausgewachsen" und ,in seine Verfallsperiode eingetreten", 2 so daß sich die Enteignung der Bauern von ihrer Parzelle „mit stets wachsender Geschwindigkeit” durchsetzte. ${ }^{3}$ Mochte es vorerst auch noch „einen tiefen Widerspruch [...] zwischen dem industriellen Proletariat und der Bauernschaft" geben, ${ }^{4}$ so stellte doch das, „was den Bauern vom Proletarier trennt, nicht mehr sein wirkliches Interesse, sondern nur noch sein illusionäres Vorurteil" dar ${ }^{5}$ - nämlich das Vorurteil darüber, daß sich der Bauer noch im vollen Besitz seiner Eigentumsrechte wähnte, obwohl er sie faktisch längst an seine Gläubiger verloren und allein die Pflicht zu Zinszahlungen behalten hatte. Wurden ihm über diese Situation die Augen geöffnet, so mußte das trennende Vorurteil bald fallen und der Verständigung mit dem Proletariat weichen.

Ganz in diesem Sinne hat Marx 1856 gegenüber Engels den Erfolg einer möglichen Revolution in Deutschland davon abhängig gemacht, ob es gelingen werde, im Ernstfalle "to back the Proletarian revolution by some second edition of the Peasants' war". MEW XXIX, S. 47 (Marx an Engels, I6. 4. 1856).

${ }^{2}$ MEW XXV, S. 8 rs; vgl. MEW XXIII, S. 784, MEW XVII, S. 55 If.

2 MEW XVII, S. 55I (1. Entwurf zum „Bürgerkrieg in Frankreich”, 1871).

3.a.O., S. 345 („Der Bürgerkrieg in Frankreich”, 1871).

4 A.a.O., S. 551 .

A.a.O., S. ssif. 
Nach den bisherigen Feststellungen hat Marx das Bündnis zwischen Arbeitern und Bauern, ungeachtet gewisser Schwierigkeiten, für notwendig und aktuell gehalten. Es bleibt aber zu untersuchen, ob die Bündnisfrage für alle Länder dieselben Formen haben oder ob sie von Fall zu Fall differenziert betrachtet werden sollte. Der ausschlaggebende Maßstab mußte für Marx der Stand der ökonomischen Entwicklung sein, nach dem sich Form und Ziel des Bündnisses zu richten hatten. Das eigentliche und letzte Ziel des Zusammenwirkens von Proletariat und Bauerntum sollte die Verwirklichung der sozialistischen Gesellschaft sein, doch die Bedingungen hierfür waren nach Marx erst an einer Stelle in vollem Umfange vorhanden: in England. Von diesem Land sagte er, es sei ,einstweilen das wichtigste Land für die Arbeiterrevolution, dazu das einzige Land, wo die materiellen Bedingungen dieser Revolution bis zu einem gewissen Reifegrad entwickelt sind." 1 Hier hätte also das Bündnis ohne weiteres die Errichtung des Sozialismus, d.h. die Beseitigung des Privateigentums an allen Produktionsmitteln, zum Ziel haben können.

Aber auch in den anderen, ökonomisch weniger weit fortgeschrittenen Ländern konnte eine Situation eintreten, die eine Klärung des Verhältnisses zwischen Arbeitern und Bauern erheischte. Marx ist auf dieses Problem in erster Linie durch die politischen Ereignisse in Frankreich hingelenkt worden. Die Revolutionen von I 848 und 1871 hatten zu erkennen gegeben, daß auch in Ländern, die ökonomisch noch nicht für den Sozialismus reif geworden waren, sich die Gelegenheit für einen politischen Sieg des Proletariats bieten könnte. Dessen Ergebnis wäre aber nicht sogleich der Übergang zur sozialistischen Gesellschaft gewesen, sondern auf ihn mußte eine Phase folgen, in der das Proletariat gemeinsam mit den anderen werktätigen Klassen die Grundlagen des Sozialismus zu schaffen hatte. Die Frage war, wie das Proletariat, einmal in den Besitz der politischen Macht gelangt, sich mit friedlichen Mitteln des Beistandes der Bauern, die gerade in Frankreich dank ihrer Menge eine ausschlaggebende Rolle spielten, versichern konnte. Die Antwort erblickte Marx darin, daß „die sozial-demoktatische, die rote Republik" als die "Diktatur der Verbündeten" des Bauern ${ }^{2}$ es in der Hand hätte, all die Lasten, die die kapitalistische Gesellschaft den Bauern auferlegt hatte, die Hypo-

${ }^{1}$ MEW XXXII, S. 669 (K. Marx an S. Meyer und A. Vogt, 9. 4. 1870). Ferner MEW XVI, S. 559 (Aufzeichnung zweier Reden von Karl Marx über das Grundeigentum, 6. 7 . r 869): „in England könne der Grund und Boden innerhalb von vierzehn Tagen durch einen Parlamentsakt in Gemeineigentum umgewandelt werden".

${ }^{2}$ MEW VII, S. 84 („Die Klassenkämpfe” usw.); vgl. MEW XVIII, S. 63 of. 
theken- und die Wucherzinsen sowie die Staatssteuern, abzuschaffen und dadurch zu beweisen, wer der uneigennützige Freund der Landbevölkerung sei. Die Schikanen des herrschenden Systems seien derart geworden, daß die siegreiche Kommune von i87I sich schnell als die „einzige Macht” bestätigt hätte, die dem Bauern „große unmittelbare Wohltaten sogar unter ihren gegenwärtigen ökonomischen Bedingungen erweisen kann", indem sie ihn von jenen Lasten befreite und zugleich den Übergang aus seinem derzeitigen Elend in einen Zustand, der ihm ein ,wirkliches Eigentum an den Früchten seiner Arbeit" gewährte, ermöglichte. ${ }^{1}$ Das waren Schritte, die noch nicht an die grundsätzliche Frage des Privateigentums heranführten, sondern zunächst eine Besserung der ökonomischen und sozialen Lage des Bauern bezweckten, um in ihm ein festes Vertrauen zur Politik des Proletariats zu erwecken. Verglichen mit der Grund- und Bodenfrage stellte das eine Politik der kleinen Mittel dar, deren Sinn es war, den Bauern davon abzuhalten, in das Lager der Reaktion, der Gegenrevolution abzuschwenken und das Proletariat der Isolierung preiszugeben.

Marx hat angenommen, daß auf dem Kontinent allein durch Beseitigung des Drucks, den die kapitalistische Gesellschaft auf die Bauern ausübte, letztere für ein Bündnis mit dem Proletariat gewonnen werden könnten. Die Möglichkeit, daß die Bauern darüberhinaus den Wunsch haben könnten, die eigentlichen Ursachen ihrer ökonomischen Schwäche, den zu geringen Landbesitz, zu beseitigen, hat er zwar nicht übersehen, aber als politisches Negativum bewertet, das die Arbeiterpartei zu umgehen habe. Er hat ausdrücklich davon abgeraten, analog dem Beispiel von 1789 das große Grundeigentum unter die Bauern aufzuteilen; ${ }^{2}$ statt dessen hat er darauf gedrungen, den Grund und Boden zu nationalisieren, da dies eine alle Eigentumsrechte entkräftende gesellschaftliche Notwendigkeit sei. ${ }^{3}$

\footnotetext{
${ }^{1}$ MEW XVII, S. 552 (1. Entwurf usw.); vgl. auch die Maßnahmen, die im Kommunistischen Manifest dem zur Herrschaft gelangten Proletariat vorgeschlagen werden, MEW IV, S. $48 \mathrm{I}$.

${ }^{2}$ MEW VII, S. 25 If. („Ansprache der Zentralbehörde an den Bund vom März 1890”): die Arbeiter hatten zu verlangen, „daß das konfiszierte Feudaleigentum Staatseigentum bleibt und zu Arbeiterkolonien verwandt wird, die das assoziierte Landproletariat [. . ] bearbeitet." Im gleichen Sinne hat sich Marx Jahrzehnte später an einer Stelle geäußert, an der er grundsätzlich für eine gewinnende Taktik des Proletariats gegenüber den Bauern eingetreten ist; er widerriet es abermals, das Parzelleneigentum „einfach durch Annexation der großen Güter an die Bauern” zu kräftigen. MEW XVIII, S. 633 (Konspekt von Bakunins "Staatlichkeit und Anarchie”, 1874).

${ }^{3}$ MEW XVIII, S. 60 („Uber die Nationalisierung" usw.).
} 
Unter diesen Voraussetzungen hat der Bündnisgedanke eine Form erhalten, die den Bauern eine ganz spezifische Rolle angewiesen hat. Marx hat erwartet, daß sie nicht als eine selbständige, sondern als eine der Arbeiterpartei sich anschließende und unterordnende politische Kraft aktiv werden würden; seiner Vorstellung nach sollten sie „das städtische Proletariat als ihren Führer und Erzieher" begrüßen, ${ }^{1}$ dem sie sich als ihrem „Vorkämpfer” und „natürlichen Verbündeten” anzuschließen hätten. ${ }^{2}$ Daraus folgt, daß es sich bei dem Bündnis nicht um den Kompromiß zwischen zwei selbständigen politischen Partnern handelte, sondern um das Zusammengehen zweier ihrem Wesen nach identischer Gruppen bzw. um die freiwillige Unterordnung der einen unter die andere. Von Marx war nicht beabsichtigt, zwei unabhängige Parteien, die ihre gemeinsamen Interessen gegenüber Dritten wahren wollten, kooperieren zu lassen, sondern ihm schwebte die Vereinigung zweier Klassen vor, die von der sich entfaltenden Identität ihrer Klassenpositionen unaufhaltsam und für immer zueinandergeführt wurden. Diese Identität, die zunächst nur immanent war, sich jedoch über kurz oder lang realisieren mußte, war für ihn das wahrhaft konstituierende Element des Bündnisses. Aufgabe der Arbeiterpartei als der führenden Kraft innerhalb des Bündnisses mußte es sein, dieses Element zum Tragen zu bringen und die Bauern von seiner Existenz zu überzeugen.

Trotz der vorausgesetzten substantiellen Harmonie zwischen Proletariat und Bauerntum lag dem Bündnis eine an Selbstentäußerung grenzende Metamorphose des letzteren zugrunde, wie sie nur von einer schwachen und unselbständigen sozialen Gruppe erwartet werden konnte, und es ist zu fragen, welche Bauern Marx in diesem Zusammenhange gemeint hat. Wie näheres Zusehen ergibt, hat er die Kleinbauern oder Parzellenbesitzer als repräsentativen Typ des Bauern benutzt ${ }^{3}$ und an ihnen die Inkongruenz zwischen bäuerlichem Grundbesitz und modernen Produktionsformen demonstriert. Die Bekanntschaft mit diesem Typ des Bauerntums dürfte Marx den Eindrücken verdankt haben, die er von den ländlichen Verhältnissen

${ }^{1}$ MEW XVII, S. 55I (I. Entwurf usw.).

2 MEW VII, S. 2 I („Die Klassenkämpfe” usw.); MEW VIII, S. 202; MEW IV, S. 472.

${ }^{3} \mathrm{MEW}$ XXV, S. 81 $2 \mathrm{ff}$ : hier handelt Marx den bäuerlichen Kleingrundbesitz expressis verbis unter dem Kennzeichen „Parzelleneigentum” ab; vgl. MEW XXV, S. 689, wo dem Bauern die „kleine Parzelle” zugeordnet wird; a.a.O., S. 882, exemplifiziert Marx auf den „kleinen Bauer"; MEW XXIII, S. 775, ebenso; ferner werden MEW XVIII, S. 633, Parzelleneigentum und Bauer einander gleichgesetzt. 
seiner moselländischen Heimat ${ }^{1}$ sowie Frankreichs gewonnen hatte ${ }^{2}$ und die einen sichtbaren Kontrast zu den Zuständen in England darstellten. Jedoch ist es fraglich, ob diese Anschauung verallgemeinert werden durfte, denn z.B. östlich des Rheins herrschten andere Bedingungen. Dort ist die soziale Schichtung der Landbevölkerung teilweise noch so vielfältig gewesen, daß mit einem Schema, das lediglich nach Parzellen- bzw. Kleinbauern, Landarbeitern und Großgrundbesitzern differenzierte, die Wirklichkeit nicht erfaßt werden konnte. Das bäuerliche Eigentum lag hier vielfach über der Grenze des bloßen Kleinbesitzes, ohne aber in kapitalistische Dimensionen hineinzureichen. Wurde die Arbeiterpartei ausschließlich auf das verelendende Kleinbauerntum als Bündnispartner hingewiesen, so wurde ihre politische Bewegungsfreiheit gegenüber den anderen bäuerlichen Gruppen von vornherein eingeengt, und die Landschaften, in denen weder ein zahlreiches ländliches Proletariat noch ein proletarisiertes Kleinbauerntum vorhanden war, mußten auf der politischen Karte der Arbeiterbewegung weiße Flecken bleiben.

\section{V}

Angesichts des Gewichts, das in der Marxschen Bündnistheorie der Annahme von der Verelendung der Bauern zukommt, ist es nicht belanglos zu prüfen, ob diese Hypothese hinreichend begründet gewesen ist oder nicht. Auszugehen ist von der Feststellung, daß es für Marx verschiedene Gründe gewesen sind, die in der Vergangenheit und Gegenwart für den Untergang des Bauerntums verantwortlich sein sollten. Im Mittelalter hatte die Landwirtschaft in den Händen von Bauern gelegen, die als Hintersassen der Grundherren zu Zins und Fronarbeit verpflichtet waren, ansonsten aber selbständig ihre Arbeit ausübten. ${ }^{3}$ Von diesem Zustand bis zum Vorherrschen des großen Pachtbetriebes führte ein Weg, auf den die Bauern etappenweise ihres Besitzes und ihrer sonstigen Existenzgrundlagen beraubt worden sind. Die Stadien des Bauernlegens, wie es sich in England seit dem I6. Jahrhundert vollzogen hat, hat Marx unter Hinweis auf die gewaltsame Vertreibung der Bauern, auf die Aneignung des Gemeindeeigentums und der ehemaligen Kirchengüter, auf die Inbesitznahme der Staatsländereien und die Abwälzung der feudalen Lehns-

1 Vgl. seine Artikel in der Rheinischen Zeitung aus dem Jahr 1843 ; MEW I, S. 172 ff.

${ }^{2}$ Mit der sozialen Lage und der politischen Rolle des Bauerntums in Frankreich hat sich Marx wiederholt beschäftigt, s. MEW VII, S. 83 ff., MEW VIII, S. 198ff., MEW XVII, S. 345, 551 .

${ }^{3}$ MEW XXIII, S. 745: „In allen Ländern Europas ist die feudale Produktion durch

Teilung des Bodens unter möglichst viele Untersassen charakterisiert". 
pflichten auf den Staat beschrieben - alles Schritte, die die feudalen Grundherren mit Erfolg unternommen hatten, um sich in den unmittelbaren Besitz des Ackerlandes zu setzen, es territorial zu erweitern und in ihr Privateigentum zu verwandeln. ${ }^{1}$ Ergänzt wurde der Vorgang durch das Aufkommen der Manufakturen, die die ländliche Heimindustrie vernichteten und damit die zweite Säule der bäuerlichen Existenz umstürzten. ${ }^{2}$ „Die Verwandlung der individuellen und zersplitterten Produktionsmittel in gesellschaftlich konzentrierte", die auf diese Weise bewirkt wurde, bezeichnete Marx als die „Vorgeschichte des Kapitals", ${ }^{3}$ d.h. als eine notwendige Entwicklung, die die allgemeinen Grundlagen einer kapitalistischen Landwirtschaft sowie das erforderliche Proletariat für die kapitalistische Industrie schuf. Das Bauerntum, das im Mittelalter kennzeichnend für die Landwirtschaft gewesen ist, wurde reduziert und großenteils in das „Kunstprodukt der modernen Geschichte", in freie Lohnarbeiter, verwandelt. ${ }^{4}$

Dieser Vorgang konnte sich im I9. Jahrhundert nicht unverändert fortsetzen, da die meisten seiner Ursachen nicht mehr bestanden. Das Bauernlegen und die Ausweitung des Großgrundbesitzes ist seinerzeit durch die Stellung der Ritter als Lehns- und Grundherren ihrer Hintersassen, durch die Schollengebundenheit und die eingeschränkten Besitzrechte der Bauern ermöglicht worden. Dieses feudalrechtliche Sozialgefüge war nun durch die bürgerlich-kapitalistische Gesellschaft abgelöst worden, in der nicht zuletzt die Unantastbarkeit des Privateigentums proklamiert worden war. Die zu Eigentümern ihres Landes gewordenen Bauern genossen den Schutz der bürgerlichen Gesetze und konnten nicht mehr durch nackte Gewalt oder durch Ausnutzung feudaler Prärogativen vertrieben werden; sie hatten allein die Gefahr mangelnder ökonomischer Konkurrenzfähigkeit zu fürchten.

Diesem veränderten Tatbestand hat Marx dadurch Rechnung zu tragen versucht, daß er den von ihm erwarteten Untergang des Bauerntums nicht mehr von den Willkürakten der Großgrundbesitzer, sondern von der Macht der ökonomischen Faktoren erwartete. Er hielt den Ruin der selbstwirtschaftenden Bauern für unvermeidlich, weil sie unfähig seien, die Konkurrenz der kapitalistischen Großkultur zu bestehen. ${ }^{5}$ Außerdem litten sie unter gewissen willkürlichen

${ }^{1}$ MEW XXIII, S. 744ff.

2 A.a.O., S. 775 f.

${ }^{3}$ A.a.O., S. 789 f., 742 .

4 A.a.O., S. 788.

${ }^{5}$ MEW XXV, S. 81 s. Die an dieser Stelle des weiteren als Verelendungsursachen angeführte Vernichtung der Hausindustrie und Usurpation des Gemeineigentums durch die Großgrundbesitzer gehören noch in die vorkapitalistische Entwicklungsphase und haben in der zweiten Hälfte des $\mathbf{1 9}$. Jahrhunderts nur eine geringe Bedeutung gehabt. 
Momenten, die ihr Dasein verkürzen oder erschweren konnten: unter dem Wucher und den Steuern. ${ }^{1}$ Mit ihnen verfügte die bürgerliche Gesellschaft über Handhaben, die ökonomisch bedingte Verelendung zu beschleunigen bzw. auf dem Wege der Zwangsbeitreibung von Schulden und Steuern zu vollenden.

Aber war von diesen Faktoren die vorausgesagte Wirkung zu erwarten? Es besteht Anlaß zur Skepsis, weil der Untergang des Bauerntums im kapitalistischen Zeitalter keine ökonomische Notwendigkeit mehr war. Wie erwähnt, hatte Marx das Bauernlegen der spätfeudalen Zeit als notwendig für die Entstehung der ökonomischen und sozialen Voraussetzungen des Kapitalismus angesehen: für die Ansammlung eines besitzlosen Proletariats, für die beginnende Konzentration der Produktionsmittel, für die Warenproduktion und für die Entfaltung des inneren Marktes. Diese Veränderungen waren nur möglich, wenn das mittelalterliche Bauerntum, das in ökonomischer Hinsicht selbstgenügsam gewesen ist, zerstört und in eine Menschenmenge verwandelt wurde, die ihre Unterhaltsmittel auf dem Markt der kapitalistischen Wirtschaft suchen mußte. Obwohl dieser Prozeß von Gewalttaten und Not begleitet worden ist, ist er für den gesellschaftlichen Fortschritt unvermeidlich und deshalb sinnvoll gewesen.

Doch wie verhielt es sich damit, wenn die kapitalistische Gesellschaft etabliert war und das noch vorhandene Bauerntum nicht mehr ihren weiteren Aufschwung hindern konnte? Dann wirkten zwar die Gesetze der kapitalistischen Wirtschaft auf den bäuerlichen Betrieb ein und konnten ihn zum Erliegen bringen, aber eine im höheren Sinne notwendige Tendenz war darin nicht zu erblicken. Diese wäre nur gegeben gewesen, wenn die in der bäuerlichen Landwirtschaft unrationell ausgenutzten Arbeitskräfte an anderer Stelle der Volkswirtschaft dringend benötigt worden wären oder wenn die Volksernährung nur durch Überführung des bäuerlichen Grundbesitzes in leistungsfähigere Großbetriebe hätte gesichert werden können. ${ }^{2}$ Während der letztgenannte Faktor bisher nicht in Kraft getreten ist, ist der erstgenannte zwar seit langem wirksam, hat aber den bäuerlichen Betrieb nicht ruinieren können. Wie wenig der etablierte Kapitalismus auf dem Verschwinden der Bauern $\mathrm{zu}$ bestehen brauchte,

${ }^{1}$ Ebda. Vgl. dazu die Ausführungen über den Druck, den Schulden und Steuern auf die französischen Bauern ausübten: MEW VII, S. ${ }_{3}$ f., MEW VIII, S. 20If., MEW XVII, S. sgif.

2 Marx hat davon gesprochen, daß die „ständig wachsenden Bedürfnisse der Bevölkerung” und das „dauernde Steigen der Preise landwirtschaftlicher Erzeugnisse” eine Nationalisierung des Grund und Bodens als notwendig erwiesen hätten - MEW XVIII, S. 60 („Uber die Nationalisierung" usw.) -, aber als zwingende Momente haben sich diese Tendenzen nicht durchgesetzt. 
erhellt daraus, daß seit dem Ausgang des 19. Jahrhunderts bürgerliche Regierungen bemüht sind, die ökonomischen Tendenzen, die der Landwirtschaft im allgemeinen und den Bauern im besonderen abträglich sind, durch ausgesuchte Maßnahmen einzuschränken. Mögen deren Zweckmäßigkeit und die hinter ihnen stehenden Motive umstritten sein, so bleibt es doch an dem, daß aus politischen Erwägungen heraus den potentiellen ökonomischen Entwicklungstendenzen entgegengetreten wird. Und das dürfte, sofern es Erfolg hat, im Sinne der marxistischen Theorie nur möglich sein, weil der definitive Untergang des Bauerntums unter der Herrschaft des Kapitalismus nicht notwendig ist, mithin der Kapitalismus in diesem Punkte nicht als der Wegbereiter des Sozialismus auftritt. Das hat zur Folge, daß die Bauern, die für Marx bereits eine vor-kapitalistische Klasse gewesen sind, nicht nur den Feudalismus, sondern auch den Kapitalismus überleben können und selbst noch beim Anbruch der sozialistischen Gesellschaft existieren. Dadurch sind die sozialistischen Arbeiterparteien genötigt worden, in der Bauernfrage eine differenziertere Haltung einzunehmen, als sie von Marx vorgeschlagen worden ist. ${ }^{1}$ Vor allen Dingen hat unter diesen Umständen berücksichtigt werden müssen, daß die Bündnisfrage kein kurzfristiges, sondern ein langfristiges Problem war, dessen Lösung nicht von einer zwangsläufigen Entwicklung herbeigeführt werden würde, sondern nur durch aktive Politik erreicht werden konnte.

Die Probe aufs Exempel dieses Teils der marxistischen Agrartheorie ist zu Lebzeiten ihres Begründers nur in begrenztem Umfange gemacht worden. Das lag daran, daß die in der zweiten Hälfte des I9. Jahrhunderts enstehenden Arbeiterparteien zunächst wenig Gelegenheit hatten, praktische Agrarpolitik zu treiben oder dauerhaften Kontakt mit der Landbevölkerung aufzunehmen. Die Agrarfrage war eine theoretische Angelegenheit, deren sich die Arbeiterparteien fürs erste mehr aus prinzipiellen denn aus praktischen Gründen annahmen. Allerdings stand dahinter die Überzeugung, daß das organisierte Proletariat bald imstande sein würde, seine theoretischen Anschauungen in der Praxis zu verwirklichen. Deshalb wurde die richtige Orientierung in der Agrarfrage mit großem Ernst gesucht.

\footnotetext{
${ }^{1}$ Dessen ist die Arbeiterbewegung auch nicht durch das Aufkommen der sog. überseeischen Konkurrenz enthoben worden, von der sich noch Friedrich Engels - s. seine Glosse im 3. Band des „Kapital”, MEW XXV, S. 735f. - und Karl Kautsky - s. F. Schaaf, S. 230 - das schnelle Ende der europäischen Landwirtschaft versprochen hatten.
} 
In diesem Zusammenhange entwickelten sich innerhalb der 1864 gegründeten Internationalen Arbeiter-Assoziation heftige Auseinandersetzungen zwischen den Anhängern Marx' und Proudhons bzw. Bakunins. Gerade die Agrarfrage ist zwischen Marxisten und Proudhonisten besonders umstritten gewesen, weil letztere den Standpunkt vertraten, daß der kleine Grundbesitz gestärkt und als Element der menschlichen Freiheit erhalten werden müsse. ${ }^{1}$ Obwohl Marx auf die öffentlichen Debatten der Internationalen über die Bodenfrage nur einen mittelbaren Einfluß genommen hat, hat er die Aktualität dieses Themas bereits in der Inauguraladresse mit dem Satz: „wenn die Konzentration des Landes in wenigen Händen gleichmäßig fortschreitet, wird sich die Grund- und Bodenfrage [...] ganz merkwürdig vereinfachen", ${ }^{2}$ anklingen lassen. Und es waren seine Ideen, die auf den Kongressen der Internationalen den Proudhonisten entgegengehalten und schließlich zum Siege geführt wurden.

Nach einem Vorspiel auf dem Lausanner Kongreß (1 867) waren es die folgenden Treffen in Brüssel und Basel (1868, 1869), die den offenen Kampf zwischen beiden Richtungen erlebten. I 868 glaubte Marx noch, aus der Ferne urteilend, darauf dringen zu sollen, daß der Kongreß es vermeide, „Beschlüsse in allgemeinen theoretischen Fragen zu fassen", ${ }^{3}$ da so eine unter dem Einfluß der Proudhonisten drohende „Blamage” am ehesten zu verhüten gewesen wäre; aber trotzdem hat schon in Brüssel das Prinzip der Nationalisierung von Grund und Boden die Mehrheit auf seiner Seite gehabt. ${ }^{4}$ Das hinderte die Unterlegenen nicht, auf dem nächsten Kongreß eine neuerliche Entscheidung zu suchen, die diesmal vom Generalrat der Internationalen vorberaten wurde. In diesem Stadium hatte Marx Gelegenheit, sich gegen die Illusionen über das Kleinbauerntum und für die Notwendigkeit der allgemeinen Vergesellschaftung auszusprechen. ${ }^{5}$ Derart vorbereitet, wiederholte sich in Basel, was im Vorjahr schon gelungen war: der Kongreß der Internationalen sprach sich mit großer Mehrheit dafür aus, das Privateigentum an Grund und Boden abzuschaffen. ${ }^{6}$ Marx, der trotz den Vorarbeiten der ,öffentlichen Schaustellung der Partei ,mit allen ihren Geschwüren' " mit gemischten Gefühlen entgegengesehen hatte, konnte in diesem Falle erleichtert

1 Uber die Stellungnahme der Internationalen in der Agrarfrage s. F. Schaaf, S. 49ff.; J. Braunthal, Geschichte der Internationale, Bd. I, Hannover I961, S. I13, 143f., 147 , I49f.; I. M. Kriwogus, S. M. Stezkewitsch, Abriß der Geschichte der I. und II. Internationale, Berlin 1960, S. $72 f$., $82 \mathrm{f} ., 94 f$.

${ }^{2}$ MEW XVI, S. 8f. („Inauguraladresse der IAA”, 1864).

${ }^{3}$ MEW XXXII, S. 598 (K. Marx an G. Eccarius und F. Leßner, Io. 9. I868).

4 F. Schaaf, S. 58 .

5 MEW XVI, S. $558 f$. (Aufzeichnung zweier Reden usw.).

${ }^{6}$ F. Schaaf, S. 6r, 64. 
darüber sein, daß der Kongreß „verhältnismäßig so gut verlaufen” war. ${ }^{1}$ Die Internationale hatte sich definitiv den marxistischen Standpunkt in der Agrarfrage angeeignet.

Doch das war nicht mehr, als eine prinzipielle Willensäußerung auf übernationaler Ebene; inwieweit dieser Wille sich in der alltäglichen Politik verkörpern konnte, lag bei den nationalen Parteien, die ihn in der Praxis zu vertreten hatten. Und hier zeigte es sich, daß manch einer Theorie und Praxis für zweierlei Dinge hielt, die er gerne voneinander getrennt sehen wollte. Ein Beispiel dafür bot die Sozialdemokratische Arbeiterpartei Deutschlands (SDAP), die, ihrer politischen Verbundenheit mit kleinbürgerlichen und kleinbäuerlichen Gruppen eingedenk, bemüht gewesen ist, einer offiziellen Stellungnahme auszuweichen und ihre Freiheit gegenüber jenen Beschlüssen zu betonen. ${ }^{2}$ Es war in erster Linie Wilhelm Liebknecht, der es vermeiden wollte, durch die förmliche Übernahme des Prinzips der Bodenenteignung Unfrieden in den eigenen politischen Anhang zu tragen. Er nahm sich deshalb das Recht zu erklären, daß es dem freien Ermessen seiner Partei überlassen sei, ob sie sich für die Baseler Beschlüsse aussprechen wolle oder nicht; ihre allgemeine Solidarität mit der Internationalen schließe nicht die bedingungslose Übernahme aller Erklärungen derselben ein. ${ }^{3}$ Auch war er der Ansicht, daß dem Beschluß von Basel fürs erste nur eine theoretische und keine politisch-praktische Bedeutung zukomme, ${ }^{4}$ was ihn wiederum darin bestärkte, keine direkten Konsequenzen für seine eigene politische Tätigkeit aus dem Beschluß zu ziehen. Das schien ihm um so richtiger, als er glaubte, daß das Proletariat, wenn es einmal die politische Macht erobern wolle, auf den Beistand oder wenigstens die wohlwollende Neutralität der Bauern angewiesen sein werde; $;^{5}$ deshalb

${ }^{1}$ MEW XXXII, S. 632 (K. Marx an Laura Lafargue, 25. 9. 1869).

${ }^{2}$ F. Schaaf, S. 94f. Zum Unterschied davon hatte der konkurrierende lassalleanische Allgemeine Deutsche Arbeiterverein (ADAV) die Baseler Beschlüsse öffentlich bejaht.

3. Wiebknecht, Die Grund- und Bodenfrage, Leipzig 1874, S. 3: „Unser Verhältnis zur Internationalen läßt uns die Entscheidung vollkommen frei". Vgl. dazu den von Marx selbst aufgestellten Satz: „Es ist Aufgabe der Internationalen Arbeiterassoziation, die spontanen Bewegungen der Arbeiterklasse zu vereinigen und zu verallgemeinern, doch nicht, ihnen irgendein doktrinäres System zu diktieren oder aufzudrängen". MEW XVI, S. I95 (Instruktion für die Delegierten des Provisorischen Zentralrats, I 867). Uber die Motive für das Zögern der SDAP s. R. Morgan, The German Social Democrats and the First International, Cambridge 1969, S. I92ff.; F. Schaaf, S. I I Iff.; F. Mehring, Geschichte der deutschen Sozialdemokratie, 2. Teil, Berlin 1960, S. $348 \mathrm{ff}$.

4 W. Liebknecht, S. 20: „Die Baseler Beschlüsse sind wesentlich theoretischer Natur und haben keinen praktischen unmittelbaren Charakter."

${ }^{5}$ W. Liebknecht, Briefwechsel mit Karl Marx und Friedrich Engels, hrsg. von G. Eckert, The Hague 1963 , S. 96 (W. Liebknecht an F. Engels, 5. 4. 1870): „Die Industriearbeiter allein können in Deutschland keine Revolution machen [. . . Die Bauern] müssen 
trat er dafür ein, bestehende politisch-taktische Vorteile nicht durch ein Bekenntnis zu inaktuellen sozialistischen Prinzipien zu gefährden.

Nichts verständlicher, als daß dieses Hinhalten den Unwillen von Marx erregte. Ihm waren es nur „Albernheit und Schwäche”, die Liebknecht an den Tag legte; wenn im Interesse der Kleinbauern gegen die Baseler Beschlüsse remonstriert werde, so solle man doch beachten, daß in Deutschland ein ausgedehntes Großgrundeigentum existiere, das man nicht mehr ,in der veralteten Manier von 1789 " beseitigen könne, ${ }^{1}$ d.h. durch Aufteilung unter die Bauern. Unbeschadet aller Kritik klingt hier die Möglichkeit an, die Baseler Beschlüsse so zu interpretieren, daß sie vorwiegend für das Großgrundeigentum gelten, das bäuerliche Eigentum aber zunächst unberührt lassen sollten. Diesen Gesichtspunkt hat Marx einige Jahre später dahingehend präzisiert, daß das zur Herrschaft gelangte Proletariat den Bauern nicht vor den Kopf stoßen dürfe, indem es die „Abschaffung seines Eigentums" fordere, sondern als erstes komme es darauf an, Maßregeln zu ergreifen, „wodurch der Bauer seine Lage unmittelbar verbessert findet, die ihn also für die Revolution gewinnen."2 Ganz ähnlich äußerte sich Friedrich Engels, der I 869 die Ansicht zu propagieren empfahl, „daß das Proletariat vorderhand kein Interesse daran habe, den kleinen Grundbezitz in Frage zu stellen."3

Demnach war es für Marx und Engels nicht ausgeschlossen, an dem prinzipiellen Inhalt der Baseler Beschlüsse taktische Abstriche vorzunehmen, um zu verhindern, daß das Kleinbauerntum irritiert und in das bürgerliche Lager getrieben wurde. Darin lag eine gewisse Rechtfertigung für Liebknecht, der auf seine Weise mit dem Problem fertig zu werden suchte. Offensichtlich ist er aber von Marx oder Engels nicht ermutigt worden, seine taktische Rücksichtnahme auf die Kleinbauern sichtbarer auszugestalten, es blieb auch keine Zeit mehr dafür, denn die Stellungnahme Liebknechts hatte seine Partei inzwischen in eine unhaltbare Position gebracht. Vom ADAV wurde sie agitatorisch bedrängt, sich die Baseler Beschlüsse zu eigen zu machen, von den alten bürgerlichen Bundesgenossen wurde sie aufgefordert, sich von jenen Beschlüssen loszusagen (denn das Lavieren Liebknechts genügte nicht, um den Argwohn dieser Kreise zu beschwichtigen), und aus den eigenen Reihen wurde eine Äußerung

wenigstens soweit gewonnen sein, daß sie uns nicht entgegentreten. Wie die Dinge jetzt liegen, würde das städtische Proletariat von den Bauern mit Dreschflegeln totgeschlagen werden."

${ }^{1}$ MEW XXXII, S. 380 (K. Marx an F. Engels, 30. ro. I869).

2 MEW XVIII, S. 633 (Konspekt von Bakunins usw.).

${ }^{3}$ MEW XXXII, S. 382 (F. Engels an K. MarX, I. I I. 1869). 
verlangt, die nicht hinter der des ADAV zurückblieb. ${ }^{1}$ So zeichnete sich eine Situation $a b$, in der sich die Partei festzufahren drohte, und es blieb nur der Ausweg, die Baseler Beschlüsse vorbehaltlos anzuerkennen und den circulus vitiosus allgemeiner Rücksichtnahme zu durchbrechen. Die Gelegenheit hierfür ergab sich auf dem Parteitag von I 870, wo August Bebel eine Resolution einbrachte und begründete, in der es hieß, daß ,die ökonomische Entwicklung der modernen Gesellschaft es zu einer gesellschaftlichen Notwendigkeit machen wird, das Ackerland in gemeinschaftliches Eigentum zu verwandeln und den Boden von Staats wegen an Ackerbaugenossenschaften zu verpachten."2

So hatte sich, von der Internationalen vermittelt, die Marxsche Auffassung von der Grund- und Bodenfrage in der deutschen Sozialdemokratie durchgesetzt. Allerdings bestand, solange die Arbeiterparteien klein und schwach waren, kaum eine Möglichkeit, diese Theorie in der Praxis der Landagitation zu erproben, so daß der Vorgang der Rezeption mehr einer Einübung in den Marxismus als einer Grundlegung der Landagitation glich. Die Internationale selbst ist nicht mehr, wie ursprünglich beabsichtigt, imstande gewesen, von ihrer grundsätzlichen Stellungnahme zu einer praktischen Betätigung in der Agrarfrage fortzuschreiten. Sie hat sich wohl 1871 die Aufgabe gestellt, „die geeigneten Mittel zur Sicherung des Anschlusses der Ackerbauer an die Bewegung des industriellen Proletariats" zu prüfen und umgehend mit der Propagierung ihrer Prinzipien auf dem Lande zu beginnen, ${ }^{3}$ aber ihr organisatorischer Verfall hat sie gehindert, eigene Erfahrungen über die agitatorische Tauglichkeit ihrer Prinzipien zu sammeln. Dadurch ist auch für Marx der unmittelbare Anlaß, die Bündnisfrage einer praktischen Antwort zuzuführen, entfallen, so daß er sie in ihrem unfertigen Zustande belassen konnte.

\section{VII}

In Karl Marx' Ausführungen über das Bauerntum und die Bündnisfrage sind theoretische und praktisch-politische Aspekte vereinigt. Während die vorwiegend theoretisch gehaltenen Komponenten dazu dienten, die langfristige Zielsetzung der Bündnispolitik zu be-

1 Vgl. R. Morgan, S. 194ff.

2 F. Schaaf, S. I 13; vgl. A. Bebel, Aus meinem Leben, 2. Teil, Berlin 1953, S. Io6f. Diese Resolution entsprach auch den grundsätzlichen Ansichten Liebknechts, die er unbeschadet seines taktisch bedingten Verhaltens bereits im Frühjaht 1870 formuliert hatte, s. W. Liebknecht, Zur Grund- und Bodenfrage, S. Goff.

3 MEW XVII, S. 42I („Beschlüsse der Delegiertenkonferenz der IAA”, 1871). Der Antrag ist von Marx eingebracht und begründet worden. 
gründen, haben die praktisch-politischen die kurzfristigen, situationsgebundenen Ziele bestimmt. Konkret heißt das, daß an Hand der allgemeinen marxistischen Theorie die sozialistische Gesellschaftsform dem Bündnis zum Ziel gesetzt wurde, daß aber daneben, entsprechend den Bedürfnissen der politischen Praxis, näherliegende, noch nicht sozialistische Ziele aufgestellt werden konnten. Marx hat versucht, die beiden Elemente in der Weise zu vereinigen, daß er - wie am französischen Beispiel gezeigt worden ist - dem Proletariat bauernfreundliche Maßnahmen vorgeschlagen hat, die sich sofort zugunsten der Bauern auswirken konnten, ohne einer sozialistischen Lösung der Eigentumsfrage vorzugreifen, d.h. sie durften weder eine Beseitigung noch eine Vermehrung des Privateigentums bewirken.

Allerdings ist zu bedenken, daß dieser Rat $187 \mathrm{I}$ erteilt worden ist, als das Pariser Proletariat sich die politische Macht vorübergehend erobert hatte und die Möglichkeit besaß, derartige Maßnahmen zu verwirklichen. Das war eine relativ günstige Position, aus der heraus Mittel, die kräftiger um die Gunst der Bauern warben, nicht mehr nötig schienen. Jedoch das ist für die Arbeiterbewegung des I9. Jahrhunderts eine Ausnahmesituation gewesen, denn im allgemeinen ist für sie die alleinige Ausübung der politischen Macht noch weit entfernt gewesen, und die Aufgabe, die tatsächlich vor ihr stand, war die, inmitten einer bürgerlich-kapitalistischen Gesellschaft das Bündnis mit den Bauern zu schaffen. Hinzu kam, daß diese Gesellschaft vielfach feudalistische und oligarchische Züge aufwies, also noch nicht demokratisch durchgebildet war und der Arbeiterbewegung nur einen begrenzten politischen Spielraum gestattete. Welche Mittel in dieser, gewissermaßen vorrevolutionären, Situation zugunsten des Bündnisses mit den Bauern eingesetzt werden sollten, hat Marx nicht gesagt, sofern man nicht den Hinweis auf die Verelendung der Bauern als eine Antwort werten will. Das bedeutete, daß die Arbeiterparteien gerade in dem aktuellsten Aspekt der Bündnisfrage sich auf ihren eigenen Einfallsreichtum verlassen oder aber auf bloßes Abwarten einstellen mußten. Es hat sich erwiesen, daß damals nicht die ökonomische oder soziale, sondern die politische, radikaldemokratische Umgestaltung des bestehenden Herrschaftssystems als nächstes vollzogen werden mußte; da der bürgerliche Radikalismus bereits stark geschwächt war, fiel der Arbeiterpartei hierbei die führende Rolle $\mathrm{zu}$, die jedoch nur ausgefüllt werden konnte, wenn es gelang, sich des Beistandes nicht-proletarischer Klassen zu versichern. Unter diesen Umständen hätte die Verständigung mit dem Bauerntum gesucht werden müssen, obwohl dieses dem sozialistischen Standpunkt möglicherweise noch ganz fern stand und nur durch Zugeständnisse an seine ökonomischen und Privateigentümerinteressen 
zu gewinnen gewesen wäre. Aus der Geschichte der Revolutionen war die Bodenreform als ein Mittel antifeudalistischer, demokratischer Politik bekannt, und sie hätte auch am Ende des r9. Jahrhunderts ein wirksames, bauernfreundliches Argument des Proletariats sein können, wenn Marx diese Maßnahme nicht ausdrücklich als unvereinbar mit dem sozialistischen Endziel abgelehnt hätte. ${ }^{1}$ Infolgedessen sind die Arbeiterparteien bei mehr oder weniger kleinen Mitteln verharrt, mit denen sie kaum die Konkurrenz der bürgerlichen Bauernagitation bestehen konnten.

Solch einem Zustand praktischer Stagnation war es gemäß, wenn das Argument an Gewicht gewann, daß die Bauern unter dem Druck des Kapitalismus zwangsläufig auf die Seite des Proletariats gedrängt werden würden. Dieser Vorgang, der durch die Lückenhaftigkeit der Marxschen Bündnistheorie und die Betonung der bäuerrlichen Misere begünstigt worden ist, war aber insofern trügerisch, als die angenommene Verelendung der Bauern keine Notwendigkeit war und die Arbeiterparteien Gefahr liefen, ihr Verhalten in der Bündnisfrage von einer nicht schlüssigen Diagnose abhängig zu machen. Dasselbe galt von der spezifischen Form des Bündnisses, die nach Marx in der Unterordnung der Bauern unter das führende Proletariat bestand und die von der erwarteten Schwächung der Bauern gerechtfertigt schien. Auch hier drohte das Vertrauen auf eine als sicher vorausgesetzte Entwicklung die Kompliziertheit der tatsächlichen Verhältnisse zu verschleiern und die Bereitschaft zu politischen Entscheidungen, ohne die das Bündnis nicht zu realisieren gewesen wäre, zu lähmen.

Das Resümee ergibt, daß das Marxsche Konzept eines Bündnisses zwischen Proletariat und Bauerntum dahin tendierte, den aus der marxistischen Theorie gewonnenen Prämissen ein Übergewicht über die praktisch-politischen Probleme zu geben. Die angeblichen Wirkungen des Kapitalismus auf das Bauerntum und die Erfordernisse der sozialistischen Zukunftsgesellschaft waren es, die - miteinander kombiniert - eine starke Zukunftsbezogenheit in das Konzept hineintrugen, dergegenüber die Aspekte der Bündnisfrage unter den aktuellen Bedingungen des ausgehenden 19. Jahrhunderts zurücktraten.

\footnotetext{
${ }^{2}$ Für Marx ist eine Vermehrung des Privateigentums selbst als taktisches, vorläufiges Argument nicht in Frage gekommen, s. MEW XVIII, S. $6_{33}$ (Konspekt zu Bakunins usw.). Dementsprechend dürfte MEW XVI, S. 568f. („Adresse det Land and Labour League an die Arbeiter und Arbeiterinnen von Großbritannien und Irland", 1869), wo neben der Nationalisierung des Bodens die innere Kolonisation, d.h. Ansiedlung der Landarbeiter und Arbeitslosen auf dem nationalisierten Boden, gefordert wurde, so zu verstehen sein, daß nicht individuelle, sondern genossenschaftliche Ansiedlung beabsichtigt war.
} 
Aus dieser Situation ergab sich für die Arbeiterparteien, die sich zum Marxismus bekannten, die Versuchung, sich auf die Prämissen des Marxschen Konzepts zu versteifen und so die Theorie zu dogmatisieren, d.h. sie der andersartigen Wirklichkeit überzuordnen und zum ausschlaggebenden Moment in einer Frage zu erheben, die nicht ohne Rücksichtnahme auf die Wirklichkeit hätte beantwortet werden können. Auf diesem Geleise hat sich die deutsche Sozialdemokratie 1895 bewegt, als sie vor einer bauernfreundlichen Agrarpolitik zurückschreckte und sich statt dessen darauf verlegte, die voraussgesagte Verelendung der Bauern abzuwarten. Darin ist zweifellos ein politisches Versagen zu erblicken, das aber auf die Diagnose des Bauerntums und auf die Vorrangigkeit der sozialistischen Prinzipien, wie sie Marx vertreten hat, zurückzuführen ist. In dem Gefühl, daß die sozialistische Umgestaltung von Wirtschaft und Gesellschaft nicht mehr fern sei, hat die SPD übersehen, daß die Bündnisfrage im kaiserlichen Deutschland eine ganz aktuelle Bedeutung hatte und daß sie nur mit dem Mut zu Entscheidungen, die den Rahmen der von Marx gutgeheißenen Maßnahmen erweitert hätten, beantwortet werden konnte. 